University of Nebraska - Lincoln

DigitalCommons@University of Nebraska - Lincoln

Faculty Publications from the Department of Engineering Mechanics

Mechanical \& Materials Engineering,

Department of

August 1997

\title{
High-frequency response of atomic-force microscope cantilevers
}

Joseph A. Turner

University of Nebraska - Lincoln, jaturner@unl.edu

Sigrun Hirsekorn

Fraunhofer Institute for Nondestructive Testing, Saarbrucken, Germany

Ute Rabe

Fraunhofer Institute for Nondestructive Testing, Saarbrucken, Germany

Walter Arnold

Fraunhofer Institute for Nondestructive Testing, Saarbrucken, Germany

Follow this and additional works at: https://digitalcommons.unl.edu/engineeringmechanicsfacpub

Part of the Mechanical Engineering Commons

Turner, Joseph A.; Hirsekorn, Sigrun; Rabe, Ute; and Arnold, Walter, "High-frequency response of atomicforce microscope cantilevers" (1997). Faculty Publications from the Department of Engineering Mechanics. 2.

https://digitalcommons.unl.edu/engineeringmechanicsfacpub/2

This Article is brought to you for free and open access by the Mechanical \& Materials Engineering, Department of at DigitalCommons@University of Nebraska - Lincoln. It has been accepted for inclusion in Faculty Publications from the Department of Engineering Mechanics by an authorized administrator of DigitalCommons@University of Nebraska - Lincoln. 


\title{
High-frequency response of atomic-force microscope cantilevers
}

\author{
Joseph A. Turner, ${ }^{\text {a) }}$ Sigrun Hirsekorn, Ute Rabe, ${ }^{\text {b) }}$ and Walter Arnold \\ Fraunhofer Institute for Nondestructive Testing, Building 37, University, D-66123 Saarbrücken, Germany
}

(Received 3 February 1997; accepted for publication 21 April 1997)

\begin{abstract}
Recent advances in atomic-force microscopy have moved beyond the original quasistatic implementation into a fully dynamic regime in which the atomic-force microscope cantilever is in contact with an insonified sample. The resulting dynamical system is complex and highly nonlinear. Simplification of this problem is often realized by modeling the cantilever as a one degree of freedom system. This type of first-mode approximation (FMA), or point-mass model, has been successful in advancing material property measurement techniques. The limits and validity of such an approximation have not, however, been fully addressed. In this article, the complete flexural beam equation is examined and compared directly with the FMA using both linear and nonlinear examples. These comparisons are made using analytical and finite difference numerical techniques. The two systems are shown to have differences in drive-point impedance and are influenced differently by the interaction damping. It is shown that the higher modes must be included for excitations above the first resonance if both the low and high frequency dynamics are to be modeled accurately. (C) 1997 American Institute of Physics. [S0021-8979(97)02115-4]
\end{abstract}

\section{INTRODUCTION}

Atomic-force microscopes (AFM) were originally developed to provide surface topography information. ${ }^{1}$ Deflection of the microscope cantilever as a function of surface location is used to generate high-resolution images of surfaces. Current applications using the AFM have moved beyond the original quasistatic motion into a dynamic range. This work has shown that vibrational excitation of the specimen surface or AFM cantilever can provide a better signal-to-noise ratio and offers opportunities for noncontact and intermittent contact methods. High-frequency contact techniques also offer the potential for high resolution measurement of material properties and surface properties such as adhesion. ${ }^{2-4}$ The high-frequency motion of an elastic cantilevered beam in contact with complex surface forces is, however, not easily described. The tip-sample interaction forces are, in general, nonlinear and are the subject of intensive research themselves. When an elastic beam interacts with these surface forces, the many flexural modes may also be a part of the dynamics. It is now recognized that these higher modes are often excited in experiments. ${ }^{5-7}$

The complexities of the elastic beam vibrations have given rise to models that simplify the dynamics considerably. In one such approach the elastic beam equation is approximated with a one degree of freedom mass-spring model. ${ }^{8-10}$ The mass and stiffness of this oscillator are chosen such that the natural frequency is the same as the natural frequency of the first flexural mode of the beam. All higher modes are thus neglected. One would then assume that this type of firstmode approximation (FMA) is only valid for surface excitation frequencies well below the second natural frequency of the beam. Higher frequency vibrations would require inclusion of the higher modes. This type of model is very effective when the cantilever is much stiffer than the specimen

\footnotetext{
${ }^{a}$ Present address: Department of Engineering Mechanics, 212 Bancroft Hall, University of Nebraska-Lincoln, Lincoln, NE 68588-0347.

${ }^{b)}$ Electronic mail: rabe@izfp.fhg.de
}

surface- a condition that assures that the measured signal is not dominated by the cantilever compliance. ${ }^{10}$ Such a model has also been used to help in further understanding of nonlinear tip-force interactions. However, the importance of the higher modes and thus the limitations of FMA have not been fully investigated. Because these higher excitation frequencies can be useful for determining material properties, this type of understanding is important.

In this article, the elastic beam equation describing the flexural vibrations is examined and compared directly with FMA using both analytical and numerical techniques. The higher modes are shown to be an important part of higher frequency dynamics. For the linear case, the drive-point impedance and the effect of interaction damping are shown to be fundamentally different in these two dynamical systems. These aspects are shown explicitly in the numerical examples presented as well. When the boundary condition is nonlinear, FMA is also shown to predict a different response than that of the elastic beam. All of these differences are shown to be important when the excitation frequency is above the first flexural natural frequency as expected.

\section{GOVERNING EQUATIONS}

The atomic-force microscope cantilever here is a cantilevered, rectangular elastic beam as depicted in Fig. 1(a). The beam has width $a$, height $b$, and length $L$. The equation that governs the flexural vibrations of such an elastic beam is given by ${ }^{11,12}$

$$
E I y^{\prime \prime \prime \prime}(x, t)+\gamma \dot{y}(x, t)+\rho A \ddot{y}(x, t)=0,
$$

where the primes or dots denote the corresponding derivatives with respect to space, $\partial / \partial x$, or time, $\partial / \partial t$. Here $\rho$ is the material density, $E$ is the elastic modulus, $A=a b$ is the cross-sectional area, and $I=a b^{3} / 12$ is the bending moment of inertia. The material damping is characterized by $\gamma$. 


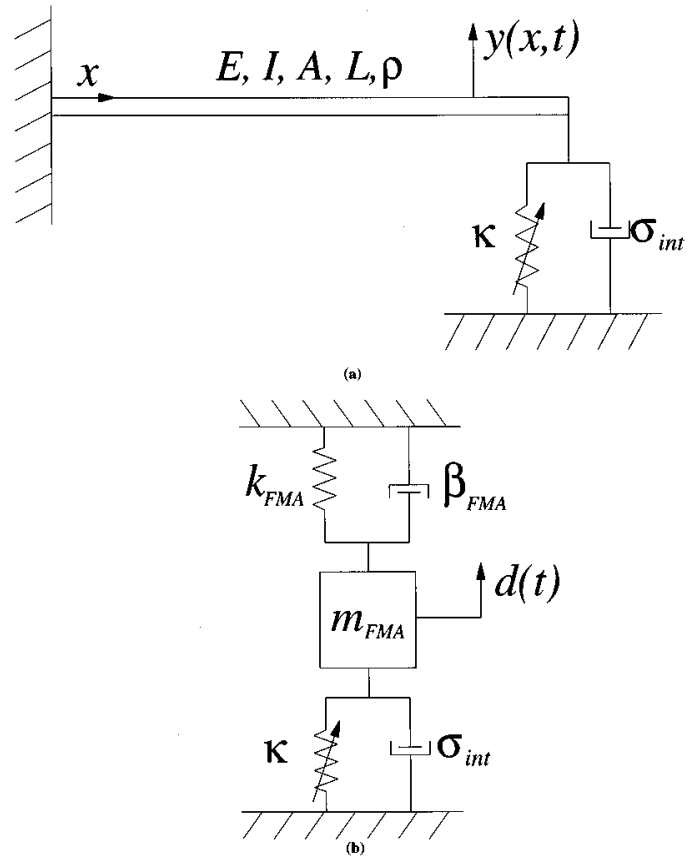

FIG. 1. Schematics of the two dynamical systems compared here. Elastic beam (a) and spring-mass system (b) coupled to a moving surface with a linear dashpot and linear or nonlinear spring.

The initial conditions considered here are given by

$$
y(x, 0)=-\frac{F_{0}}{6 E I} x^{2}(x-3 L), \quad \dot{y}(x, 0)=0 .
$$

Thus, the above formula for the initial deformation gives the initial deflection due to an end load $F_{0}$ defined as being positive when it acts in the upward direction. The initial velocity of the beam is assumed here to always be zero.

Boundary conditions must also be specified. At the cantilevered end, the boundary conditions are

$$
y(0, t)=a_{1}(t)=A_{1} \sin \left(\Omega_{1} t\right), \quad y^{\prime}(0, t)=0,
$$

such that the displacement is equal to a harmonic loading and the slope is equal to zero. Often this end will be assumed to be clamped such that $A_{1}=0$.

The other end of the beam, $x=L$, is assumed to be interacting with the surface through surface forces. Thus the moment and shear force at this end of the beam are prescribed. These boundary conditions are written

$$
\begin{aligned}
& y^{\prime \prime}(L, t)=0, \\
& E I y^{\prime \prime \prime}(L, t)=-F_{s}\left[y(L, t), a_{2}(t)\right] \\
& +\sigma_{\mathrm{int}}\left[\dot{y}(L, t)-\dot{a}_{2}(t)\right], \\
& a_{2}(t)=A_{2}(t) \sin \left(\Omega_{2} t\right) .
\end{aligned}
$$

The function $F_{s}$ represents any type of elastic force displacement relation, linear or nonlinear, between the end of the beam and the surface. Its dependence on displacement, velocity, and other parameters will be addressed when needed. This force relation is represented in Fig. 1 as the spring with spring constant $\kappa$ which is not necessarily linear. Vibration of the specimen surface is described by the function $a_{2}(t)$ assumed here to be harmonic with time dependent amplitude,
$A_{2}(t)$. The interaction damping, characterized by $\sigma_{\text {int }}$, is assumed here to be a linear, viscous type of damping.

The equation of motion, Eq. (1), with appropriate initial conditions and boundary conditions, can be solved analytically provided the shear boundary condition function $F_{s}$ is simple enough and linear. ${ }^{11,12}$ If $F_{s}$ is nonlinear, as is the case for most tip-surface interaction models, ${ }^{7,10}$ the solution of Eq. (1) is not trivial.

One approach often used to simplify the equation of motion is to approximate the motion of the end of the beam by an equivalent mass-spring system. ${ }^{8-10}$ Such a system is shown in Fig. 1(b). The natural frequency of this mass-spring oscillator is chosen to equal the natural frequency of the first flexural mode. This type of FMA, often referred to as the point-mass model, is a low-frequency approximation. The motion of the end of the beam, $d(t) \cong y(L, t)$, is in this case characterized by

$$
\begin{aligned}
m_{\mathrm{FMA}} & \ddot{d}(t)+2 m_{\mathrm{FMA}} \beta_{\mathrm{FMA}} \dot{d}(t)+k_{\mathrm{FMA}} d(t) \\
= & F_{s}\left[d(t), a_{2}(t)\right]-\sigma_{\mathrm{int}}\left[\dot{d}(t)-\dot{a}_{2}(t)\right] .
\end{aligned}
$$

The mass, spring stiffness, and damping in Eq. (5) are related to the parameters of the beam and given by

$$
\begin{aligned}
& m_{\mathrm{FMA}}=\frac{3 \rho A L}{(1.875)^{4}}, \\
& k_{\mathrm{FMA}}=\frac{3 E I}{L^{3}}, \quad 2 \beta_{\mathrm{FMA}}=\frac{\gamma}{\rho A} .
\end{aligned}
$$

Equation (5), though much simpler, retains the complication of a possible nonlinear $F_{s}$. The FMA is thus a lowfrequency approximation of the elastic beam equation. The limitations of this approximation in terms of frequency range, damping, and nonlinear behavior have not been fully investigated. Here, a number of analytical and numerical examples will be presented for both linear and nonlinear surface interactions that helps sort out these questions. The higher flexural modes will be shown to be quite important when the excitation frequency is above the frequency of the first mode.

\section{LINEAR RESPONSE}

An examination of the linear response of the elastic beam equation in comparison with the first-mode approximation is now presented. Two issues are discussed regarding the linear response of these dynamical systems described by Eqs. (1) and (5). Differences in the drive-point impedance of each system are first examined. It is shown that the FMA will always underestimate the total excitation energy of the beam when the driving frequency is higher than the natural frequency of the first flexural mode. Differences in the effect of interaction damping on both systems are then discussed. For the elastic beam interaction damping has a different effect on the higher frequency modes than the internal damping of the beam or the damping caused by losses to air. These differences can not be captured by the FMA. Finally, numerical examples that highlight both of these differences are given. 


\section{A. Drive-point impedance}

One important distinction between the elastic beam equation, Eq. (1), and the first-mode approximation, Eq. (5), is the difference in their drive-point impedances. ${ }^{12}$ The drive-point impedance, defined as the ratio of the system velocity at a particular location to the force applied at the same location, characterizes the ability of the forcing to excite the beam. When examined as a function of frequency with constant forcing amplitude, it provides a sense of how much energy the beam is willing to accept from an excitation at a particular frequency. This type of information is important for understanding the expected amplitude of excitation of the beam. In particular, predictions about the driving amplitudes necessary to cause the tip-surface separation cannot be made without accurate knowledge of the amount of energy exciting the beam. The drive-point impedance of the elastic beam will now be compared with the impedance calculated using the first-mode approximation.

A somewhat simpler equation of motion is considered to highlight this difference. The elastic beam, with damping neglected, is cantilevered at one end and free at the other. A harmonic load is applied at position $x=x_{0}$. The equation of motion is thus given by

$$
E I y^{\prime \prime \prime \prime}(x, t)+\rho A \ddot{y}(x, t)=F \delta\left(x-x_{0}\right) e^{i \omega t},
$$

where the generalized function $\delta(z)$ is infinity when its argument is zero and is zero otherwise. Equation (7) is solved by superposition using the natural modes of the unforced system. Thus, the displacement is expanded as

$$
y(x, t)=e^{i \omega t} \sum_{n=1}^{\infty} P_{n} Y_{n}(x)
$$

where the weighting of the modes, given by the coefficients $P_{n}$, is determined from the forcing. The spatial eigenfunctions, $Y_{n}(x)$, determined from the free vibration analysis of the clamped-free beam, are given by ${ }^{11}$

$$
\begin{aligned}
Y_{n}(x)= & \left(\frac{\sin \left(k_{n} L\right)-\sinh \left(k_{n} L\right)}{\cos \left(k_{n} L\right)+\cosh \left(k_{n} L\right)}\right)\left[\sin \left(k_{n} x\right)-\sinh \left(k_{n} x\right)\right] \\
& +\left[\cos \left(k_{n} x\right)-\cosh \left(k_{n} x\right)\right] .
\end{aligned}
$$

Here, $k_{n}$ are the flexural wave numbers which are related to their corresponding natural frequencies through the dispersion relation $k_{n}^{4}=\omega_{n}^{2} \rho A / E I$. The allowable flexural wave numbers, determined by the boundary conditions, satisfy the relation $^{11}$

$$
\cos \left(k_{n} L\right) \cosh \left(k_{n} L\right)=-1 .
$$

The assumed form for the displacement, Eq. (8), is substituted into the equation of motion giving

$$
\sum_{n=1}^{\infty}\left[E I k_{n}^{4}-\rho A \omega^{2}\right] P_{n} Y_{n}(x)=F \delta\left(x-x_{0}\right)
$$

The constants $P_{n}$ are then determined by multiplying Eq. (11) by $Y_{m}(x)$ and integrating from 0 to $L$. The orthogonality condition,

$$
\int_{0}^{L} Y_{m}(x) Y_{n}(x) d x=L \delta_{m n},
$$

allows the integrations to be easily evaluated. We find that

$$
P_{n}=\frac{F}{M} \frac{Y_{n}\left(x_{0}\right)}{\omega_{n}^{2}-\omega^{2}}
$$

where $M=\rho A L$ is the total mass of the beam and $\omega_{n}$ are the natural frequencies of the free beam determined from the above dispersion relation and Eq. (10). The motion of the beam to the harmonic excitation located at $x=L$ is thus

$$
y(x, t)=\sum_{n=1}^{\infty} \frac{F e^{i \omega t} Y_{n}(L) Y_{n}(x)}{M\left(\omega_{n}^{2}-\omega^{2}\right)} .
$$

From the definition of the drive-point impedance, $Z(\omega)$ $=\dot{y}(L, t) / F(t)$, we find that for the elastic beam

$$
Z_{\text {beam }}(\omega)=\frac{i \omega}{M} \sum_{n=1}^{\infty} \frac{\left[Y_{n}(L)\right]^{2}}{\left(\omega_{n}^{2}-\omega^{2}\right)} .
$$

The impedance goes to infinity at each of the natural frequencies which means that the system is very easy to excite at these frequencies. In reality, any damping present reduces these poles to a large finite value. The drive-point impedance for the first-mode approximation to the same forcing is simply

$$
Z_{\mathrm{FMA}}(\omega)=\frac{i \omega}{m_{\mathrm{FMA}}}\left(\frac{1}{\omega_{1}^{2}-\omega^{2}}\right) .
$$

From the definition of the point mass given by Eq. (6), we know that $m_{\mathrm{FMA}}=3 M /(1.875)^{4} \approx M / 4$. Using the definition of the natural modes, Eq. (9), at $x=L$, along with the condition given by Eq. (10) it can be shown that $\left[Y_{n}(L)\right]^{2}=4$. Thus, the drive-point impedance for the elastic beam is approximately equal (within 3\%) to the input impedance for the FMA plus the impedance resulting from the higher modes. In other words

$$
Z_{\text {beam }}(\omega)=Z_{\mathrm{FMA}}(\omega)+\sum_{n=2}^{\infty} \frac{4 i \omega}{M\left(\omega_{n}^{2}-\omega^{2}\right)} .
$$

Of course the FMA cannot predict the high impedances that occur at the resonances of the higher modes since these modes are neglected from the outset. However, we also see from Eq. (17) that the FMA will underestimate the drivepoint impedance for all frequencies higher than the lowest mode even away from the resonances. This result is illustrated in Fig. 2. The drive-point impedances given above were divided by $4 i \omega / M$ and squared. The logarithm of this value is shown in Fig. 2, plotted versus excitation frequency nondimensionalized by the first natural frequency. A small amount of damping was added to remove the singularities at the resonances. The drive-point impedance of the elastic beam, shown as a solid line, has many peaks at the resonances. The lower dashed line is the drive-point impedance for the FMA. It has a single peak at the first resonance followed by a decay proportional to $1 / \omega^{4}$ [corresponding to the $1 / \omega$ decay of $\left.Z_{\mathrm{FMA}}(\omega)\right]$. The smoothed trend of the elastic beam drive-point impedance is also plotted as the upper 


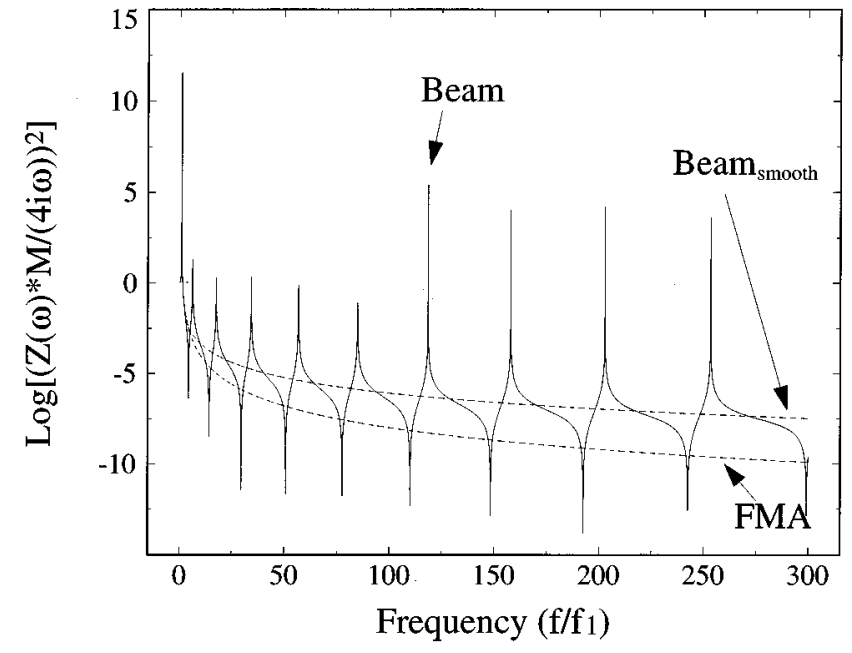

FIG. 2. Quantity related to the drive-point impedance plotted vs frequency normalized by the first natural frequency. The solid line is the drive-point impedance of the elastic beam given by Eq. (17). The upper dashed line is the smoothed trend of the elastic beam impedance which passes through the inflection points of the unsmoothed impedance. The lower dashed line is the drive-point impedance for the first-mode approximation given by Eq. (16).

dashed line. This smoothed trend, which passes through the inflection points of the nonsmoothed curve, is always higher than the FMA result. The decay of the smoothed trend is proportional to $1 / \omega^{3}$ [corresponding to the $1 / \sqrt{\omega}$ decay of $\left.Z_{\text {beam }}(\omega)\right] .{ }^{13}$ Thus for low frequencies the FMA has approximately the correct impedance as expected. However, when the excitation is above the second resonance, the difference in impedance is appreciable. If predictions are to be made about effects related to the amplitude, such as when tipsurface separation occurs, the amount of energy input into the beam must be modeled accurately. This difference in drive-point impedance will also be seen in the numerical examples presented here.

\section{B. Interaction damping effects}

The damping of vibrating atomic-force cantilevers is caused by two very different effects. System damping is the damping caused by internal losses in the beam as well as losses to any surrounding fluid in which the beam vibrates. There is also damping caused by the interaction forces which act through the contact between the cantilever tip and the specimen surface. This damping is thought to be caused primarily by adhesion effects. Uncoupled cantilevers vibrating in air have been shown to have very small damping with $Q$ varying between 90 and $900 .^{6}$ Thus, the interaction damping is expected to be the main source of damping in the coupled systems. Understanding how the interaction damping affects the beam vibration is therefore important.

In the first-mode approximation the interaction damping is modeled in the same way as the system damping of the uncoupled beam. The two dashpots are connected in series with one another as shown in Fig. 1(b). The interaction damping for the elastic beam is a boundary condition which affects the motion of the beam very differently than the damping in the beam.
This effect can be seen by solving the elastic beam free vibration problem $\left[A_{1}=0, A_{2}(t)=0\right]$ with attached linear spring and linear dashpot located at the end of the beam. This type of boundary condition has been examined previously. ${ }^{5,6}$ The predicted response was plotted as a function of frequency for different values of linear spring stiffness. ${ }^{6}$ However, the influence of the linear dashpot constant has not yet been systematically examined.

The equation of motion for this example is

$$
E I y^{\prime \prime \prime \prime}(x, t)+\rho A \ddot{y}(x, t)=0,
$$

with boundary conditions

$$
\begin{aligned}
& y(0, t)=0, \quad y^{\prime}(0, t)=0, \\
& y^{\prime \prime}(L, t)=0, \quad E I y^{\prime \prime \prime}(L, t)=\kappa y(L, t)+\sigma_{\text {int }} \dot{y}(L, t),
\end{aligned}
$$

where $\kappa$ is the spring constant of the attached linear spring $\left[F_{s}=-\kappa y(L, t)\right]$ and $\sigma_{\text {int }}$ is the interaction damping coefficient. Substitution of a general solution of Eq. (18) into the boundary conditions, Eqs. (19), results in an equation that determines the allowable values for the wave numbers. This equation is 5,6

$$
\begin{aligned}
&(k L)^{3} {[1+\cos (k L) \cosh (k L)] } \\
& \quad=\left[i p(k L)^{2}+q\right][\cos (k L) \sinh (k L)-\sin (k L) \cosh (k L)],
\end{aligned}
$$

where the above two dimensionless measures of the interaction damping, $p$, and stiffness, $q$, are

$$
p=\frac{3 \sigma_{\mathrm{int}}}{(1.875)^{2} m_{\mathrm{FMA}} \omega_{1}}, \quad q=\frac{3 \kappa}{k_{\mathrm{FMA}}} .
$$

The solution of the above transcendental equation, Eq. (20), for dimensionless wave numbers, $\xi=k_{n} L$, for general $p$ and $q$ gives a complex value. To understand what meaning these complex wave numbers have, the displacement is expanded as a superposition of modes which have the following form:

$$
\begin{aligned}
y(x, t)= & e^{i \omega t} \sum_{n=1}^{\infty}\left[B_{1 n} e^{i k_{n} x}+B_{2 n} e^{-i k_{n} x}+B_{3 n} e^{k_{n} x}\right. \\
& \left.+B_{4 n} e^{-k_{n} x}\right],
\end{aligned}
$$

where the $B \mathrm{~s}$ are again determined from the boundary conditions. Each mode of the solution given by Eq. (22) is composed of two harmonic components and two evanescent components. The first two terms are harmonic left and right traveling waves. The other two terms are left and right evanescent waves that decay exponentially in space. When the wave number is complex, the harmonic traveling waves are augmented by a spatial decay. The evanescent waves, in contrast, acquire a harmonic component. The real part of the roots found from Eq. (20) corresponds to the stiffness of the harmonic modes and thus the wave speed and frequency. The imaginary part governs the damping of the mode.

Equation (20) was solved using Mathematica ${ }^{14}$ for the complex roots $\xi=k_{n} L$ corresponding to the eigenmodes of the system. One example is illustrated in Fig. 3. A dimensionless stiffness of $q=30$ was used. The real and imaginary parts of the first three complex roots of Eq. (20) are plotted as a function of interaction damping characterized by the 


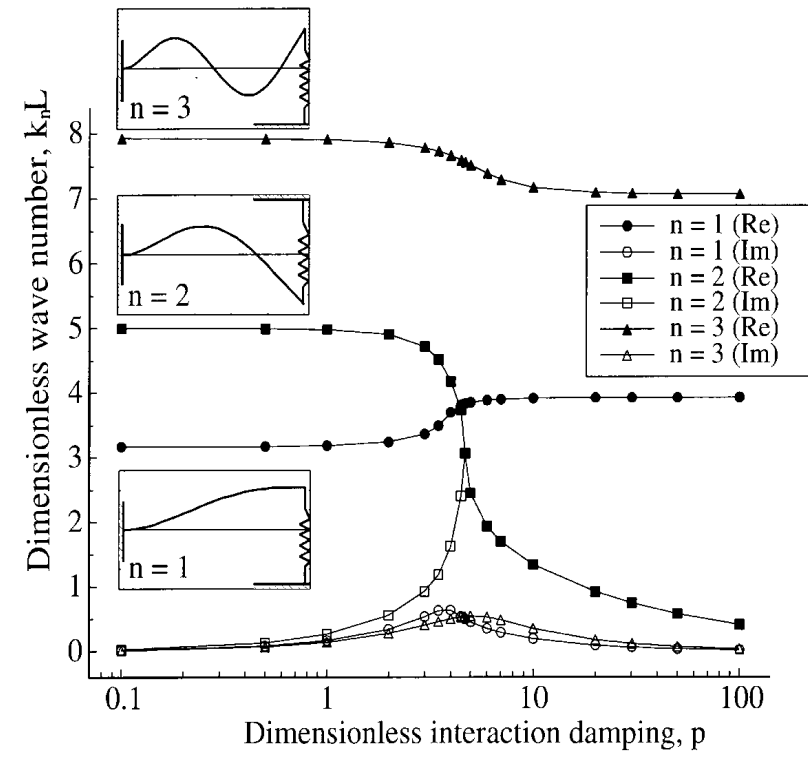

FIG. 3. Real and imaginary parts of the first three wave numbers calculated from Eq. (20) as a function of the dimensionless interaction damping, $p=3 \sigma_{\text {int }} /(1.875)^{2} m_{\mathrm{FMA}} \omega_{1}$. The dimensionless interaction stiffness is $q=3 \kappa / k_{\mathrm{FMA}}=30$. The real and imaginary parts of the second mode are seen to converge when $p$ is about 4.5. They are equal for all higher values of $p$.

dimensionless quantity, $p$. The mode shapes of these three modes without interaction damping are also shown. The results illustrate the three possible effects the interaction damping can have on the modes. We see that the first mode has an increase in the real part as the damping is increased. This mode acquires an additional stiffness from the interaction damping. The imaginary part increases until $p$ is about 4 and then begins decreasing. This mode becomes more heavily damped below a certain level of interaction damping. The damping then decreases as the interaction damping is increased further. Mode three is affected in the opposite way. As the interaction damping is increased, it is softened-its real part decreases. The damping also increases below a certain level and then decreases. The effects seen in modes one and three, caused by the interaction damping, are determined by the different mode shapes. For moderate levels of interaction damping, the mode is increasingly damped. However, once the damping becomes too large, the displacement at the end of the beam becomes much smaller, such that the dashpot cannot cause further damping. The third possible effect of damping is illustrated by mode two. This mode is also softened by increased damping. In this case, as the damping is increased, the real and imaginary parts of mode two converge. After a threshold level of interaction damping is surpassed, the real and imaginary parts are identical. The mode becomes very soft and very heavily damped. This mode passes from a state of normal damped vibration, called an underdamped vibration, into an overdamped state. ${ }^{11}$ The condition of overdamping has the effect that the mode can no longer oscillate-it is damped completely away before it can complete one period of oscillation. This overdamping effect may possibly be the reason that some modes are very difficult to detect in experiments. ${ }^{6}$ Similar results are obtained for other values of $q$ as well. One mode was always observed to behave in the same way as mode two in the example. Modes below this special mode are stiffened and those above it are softened with increasing $p$.

As seen in Fig. 3, each mode has a different sensitivity not only to the interaction stiffness but also to the tip-sample damping. For given nominal interaction stiffness and damping some modes will have a larger change in natural frequency for fluctuations in stiffness caused by moving the probe to different locations on the specimen. Therefore we would like to predict the change in wave number for changes in interaction stiffness and damping so that the optimum mode and corresponding excitation frequency can be chosen for experiments. The slope of the real part curves shown in Fig. 3 governs the change in stiffness and, therefore, natural frequency for changes in interaction stiffness and damping. The slope of the imaginary part curves governs the change in modal damping expected for changes in interaction stiffness and damping. Thus, if nominal interaction stiffness and damping properties can be approximated, the mode that should be most sensitive to changes in stiffness and damping as the probe is moved can then be determined. Those modes with the highest slopes will be most sensitive.

It has been shown in this discussion that each mode is affected very differently by the localized interaction damping because each mode has a very different mode shape. This damping is in contrast to the damping in the beam in which all modes have damping proportional to $\gamma$. The type of complicated interaction damping shown here for the higher frequencies cannot be captured by the FMA. It was also shown that the interaction stiffness and interaction damping can both lead to stiffening of the modes. This coupling effect may make it difficult experimentally to separate the effects due to interaction stiffness and interaction damping. The difference between the effect of interaction damping and beam damping is also illustrated in the numerical examples presented in Sec. III C.

\section{Temporal response}

The differences in the linear response of the elastic beam and the first-mode approximation can be clearly seen by numerical solution of the two differential equations. A finite difference solution to both equations is used to illustrate these differences. This numerical method is briefly discussed in the Appendix as well as in a number of texts. ${ }^{15,16}$ As a first check on the finite difference solution for the elastic beam, the free vibration response was calculated. Parameters typical to those found in atomic-force microscopes previously examined were used. ${ }^{5-7}$ The elastic modulus and density for $\langle 100\rangle$ silicon, $E=169 \mathrm{GPa}, \rho=2330 \mathrm{~kg} / \mathrm{m}^{3}$, respectively, were used. The beam has width $a=51$ $\mu \mathrm{m}$, thickness $b=1.5 \mu \mathrm{m}$, and length $L=262 \mu \mathrm{m}$. These values yield a first natural frequency of $f_{1}=30 \mathrm{kHz}$ $\left(m_{\mathrm{FMA}}=1.13 \times 10^{-11} \mathrm{~kg}, k_{\mathrm{FMA}}=0.404 \mathrm{~N} / \mathrm{m}\right)$. The code was run on a Digital VAX workstation. Run times were on the order of 2500 time steps per second.

For this check, the undamped beam was initially undeflected with zero initial velocity. Ninety beam segments were used such that the segment size was about 19 times smaller than the wavelength of the 10th eigenmode. The time interval was $\Delta t=2 \mathrm{~ns}$. The beam was excited at the cantilevered 


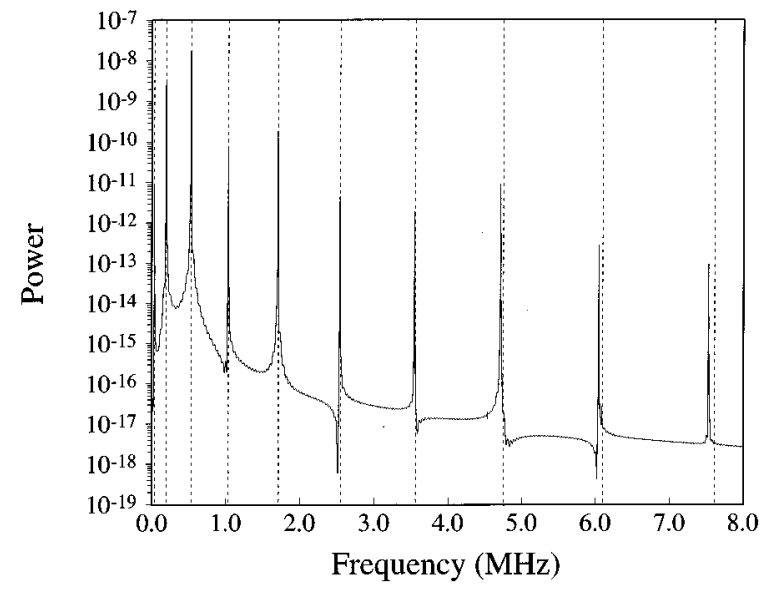

FIG. 4. Power spectrum of free elastic beam calculated using the finite difference method. The vertical dashed lines mark the theoretical flexural natural frequencies.

end with a pulse of $\Omega_{1}=500 \mathrm{kHz}$, duration of 1 cycle $(2 \mu \mathrm{s})$, and amplitude $A_{1}=2 \mathrm{~nm}$. The beam was then allowed to vibrate for $500 \mu \mathrm{s}$. The time trace of the deflection of the end of the beam was then fast Fourier transformed and squared giving a quantity proportional to the power spectrum. The result is shown in Fig. 4, up to a frequency of $8 \mathrm{MHz}$. The frequency resolution is $1900 \mathrm{~Hz}$. The vertical dashed lines are the theoretical values of the natural frequencies calculated from the above material parameters. The agreement is very good. The error in frequency of the 10th mode (7.6 $\mathrm{MHz}$ ) is $1.1 \%$. Thus for the frequency range considered here, below $5 \mathrm{MHz}$, the results should be quite good. Although the linear response of the FMA was also examined, the results are not presented here. The FMA spectrum consists of only the single peak associated with the first natural frequency. Satisfied that the numerical model accurately captures the high-frequency motion, the linear effects discussed above were then examined.

The above discussion of drive-point impedance and interaction damping are demonstrated with a simple numerical example using a linear spring with stiffness of $\kappa=790 k_{\text {FMA }}$ (linear approximation of the nonlinear function discussed in Sec. IV) and linear dashpot. The specimen surface was excited at $\Omega_{2}=1.6 \mathrm{MHz}$ harmonically. This frequency is centered roughly between the neighboring resonances of 1.38 and $1.99 \mathrm{MHz}$. The amplitude was ramped up from zero to $0.1 \mathrm{~nm}$ in 10 cycles $(6.25 \mu \mathrm{s})$ and held constant for 10 more cycles. The forcing was then stopped and the system was allowed to vibrate for $250 \mu$ s. These time domain solutions of both the elastic beam and the FMA, shown in Figs. 5(a) and 5(b), highlight the difference in drive-point impedance. As discussed above, the FMA will always underestimate the amount of energy input into the system for excitation frequencies greater than the first natural frequency. This has a profound influence on the coefficient of restitution, discussed previously, ${ }^{17}$ when the tip impacts the surface. The vibration amplitude calculated from the FMA is always lower than that found from the elastic beam solution.

The different effects from the interaction damping are also seen in Fig. 5. For the results shown, the system damp-
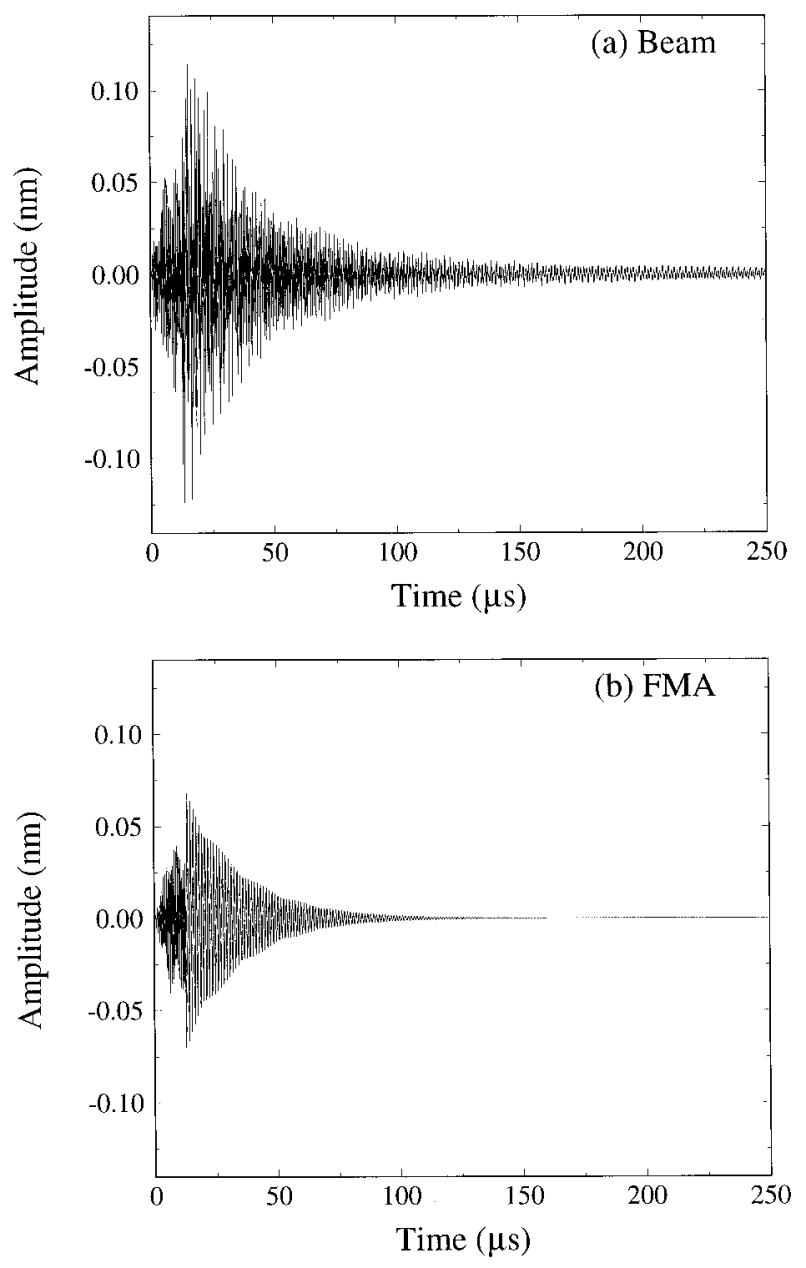

FIG. 5. Time response for both elastic beam solution (a) and first-mode approximation (b) with interaction damping only $(p=0.4)$.

ing was equal to zero $(\gamma=0)$ and the interaction damping was chosen such that $p=0.4$. The FMA solution, shown in Fig. 5(b), damps out very quickly because of the high level of damping. The elastic beam solution, shown in Fig. 5(a), has a component still remaining at the end of the time window shown. The remaining modes feel the effects of the interaction damping much less than the first flexural mode as was seen in Fig. 3. Because the interaction damping is typically much larger than the system damping, this effect is indeed important.

The different effects of the two types of damping are seen more dramatically in another example. In the first case, the system damping in each system was set equal with $\gamma / \rho A \omega_{1}=0.4$ and the interaction damping was set to zero. The second case corresponds to the results given in Fig. 5 in which the interaction damping was chosen such that $p=0.4$ and the system damping was set to zero. The results for both cases are shown in Fig. 6 which is a plot of the log square amplitude versus time. Figures 6(a) and 6(b) are, respectively, the solutions of the FMA and elastic beam for system damping only. The expected exponential decay of both solutions is apparent. The elastic beam solution has more highfrequency components, but all modes are seen to be damped proportionally. The solution for the interaction damping 


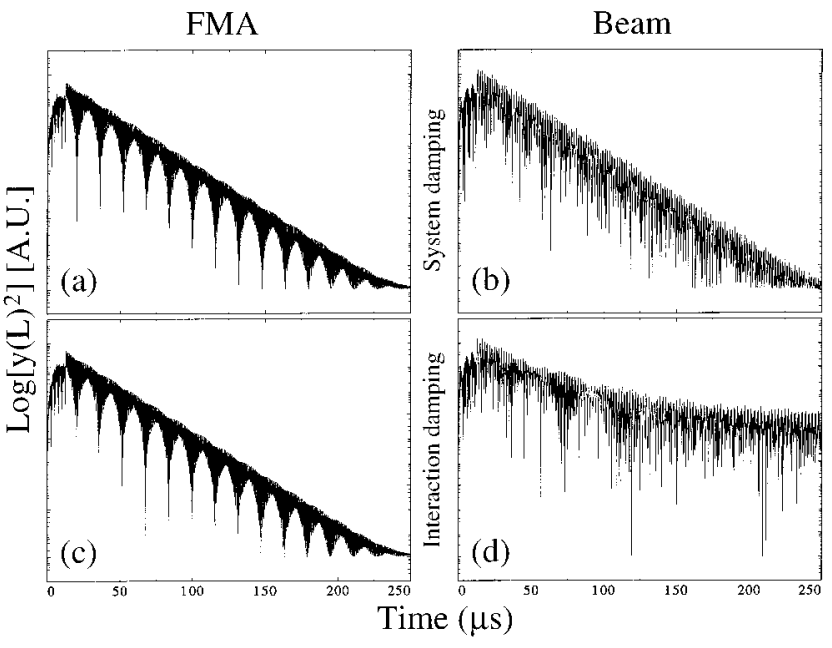

FIG. 6. Square of amplitude plotted vs time for four cases: (a) First-mode approximation with system damping only $\left(2 \beta_{\mathrm{FMA}} / \omega_{1}=0.4\right)$, (b) elastic beam solution with system damping only $\left(\gamma / \rho A \omega_{1}=0.4\right)$, (c) FMA with interaction damping only $(p=0.4)$, (d) elastic beam solution with interaction damping only $(p=0.4)$.

alone is shown in Figs. 6(c) and 6(d). The FMA response is almost the same as before, the difference resulting from the fact that the excitation has acted through the dashpot in the second case. The decay in both cases is exponential and identical as expected. The beats seen in both FMA responses, Figs. 6(a) and 6(c), occur at the frequency of the first-mode resonance. The decay of the elastic beam solution for interaction damping alone is very different. The decay begins exponential, but then becomes nonexponential after a certain amount of time. This type of nonexponential decay is characteristic of nonproportionally damped modes. ${ }^{18-20}$ Each mode has a different response at $x=L$ where the dashpot is located and is therefore damped in a different way. The modes with large amplitude motion near the end of the beam are damped most heavily and last for only a short time. This aspect of the dynamics, important for understanding surface damping properties, cannot be appropriately modeled by the FMA. Although a linear, viscous type of damping was used in this example, the outcome was a result of the damping being local. This difference in the damping is expected to be present for any type of nonlinear interaction damping as well.

Thus far we have shown how important the higher modes of vibration are for excitations above the first natural frequency. Accurate determination of the energy input into the system and for modeling the interaction damping are very much dependent upon the higher modes. The importance of these modes has, at this point, been discussed for the linear case only. In Sec. IV, the nonlinear aspects of the higher frequency modes will be shown to be equally as important to the dynamics of a vibrating elastic beam.

\section{NONLINEAR RESPONSE}

In Sec. III, the differences in the linear response of the elastic beam and the first-mode approximation were discussed. Those results highlighted the fundamental differ- ences between these two very different dynamical systems. Here the finite difference method will be used again to investigate the importance of the higher modes of vibration when the interaction forces are nonlinear. In order for a comparison to be made between the elastic beam equation and the FMA, an appropriate nonlinear interaction forcedisplacement model is needed. This force-displacement model is discussed briefly in the next section. Numerical results for the temporal response and frequency response are then presented.

\section{A. Interaction force curve}

In order to examine the effects of the higher modes on the nonlinear response in detail, a suitable nonlinear interaction force curve is needed. The goal here is to determine the degree to which nonlinear effects are not captured by the first-mode approximation. Therefore a number of nonlinear models is suitable. The model used here is discussed at length elsewhere, ${ }^{21}$ but is briefly outlined here. The interested reader is referred to the original work for a more complete discussion.

The vibrating cantilevers considered here typically have a tip at or near the end of the beam. It is through this tip that the surface interaction forces act. However, the point of the tip is not necessarily the center of action of the interaction forces. Some of the interaction forces have rather long range effects such that there is some point, or distribution of points, below the end of the beam through which the interaction forces are presumed to act. Here it is assumed that the action of the forces takes place through a single point $y(L, t)$ $-y_{0}$, such that $y_{0}$ is the location below the end of the beam that is the center of action of the forces. The center of action of the forces will be called the sensor tip here although it is recognized that it is not necessarily the tip itself.

Four different interaction force functions are used to model the four different regions of interaction forces assumed by this model. When the center of action of the forces is in contact with the specimen surface, a modified Hertzian contact model is used. In the region where the tip is interacting only with the thin water layer, the force is modeled using an adhesion model. If the sensor tip is not within the water layer, the force is assumed to be zero. The transition zone between the contact region and the adhesion layer is modeled using an interpolation that is based upon metallic adhesion effects. These different force relations are summed up as ${ }^{21}$

$$
F_{s}[z]=\left\{\begin{array}{c}
F_{\text {cont }}(z) \\
F_{\text {inter }}(z) \\
F_{\mathrm{WF}}(z) \\
0
\end{array}\right\} \text {, when }\left\{\begin{array}{c}
z \leqslant 0 \\
0 \leqslant z \leqslant z_{a} \\
z_{a} \leqslant z \leqslant 2 d \\
z \geqslant 2 d
\end{array}\right\} \text {, }
$$

where $F_{\text {cont }}, F_{\text {inter }}$, and $F_{\mathrm{WF}}$ are the contact force, the interpolating force, and the force of the waterfilm, respectively:

$$
\begin{aligned}
& F_{\text {cont }}(z)=\frac{4}{3} E^{*} \sqrt{R(-z)^{3}}-\sqrt{2 \pi \gamma_{S O} E^{*}(-R z)^{3 / 2}}+F_{0}, \\
& F_{\text {inter }}(z)=-C_{1} e^{-\alpha z}-C_{2} \\
& F_{\mathrm{WF}}(z)=-\frac{4 \pi R \gamma_{w}}{1+z / d}
\end{aligned}
$$




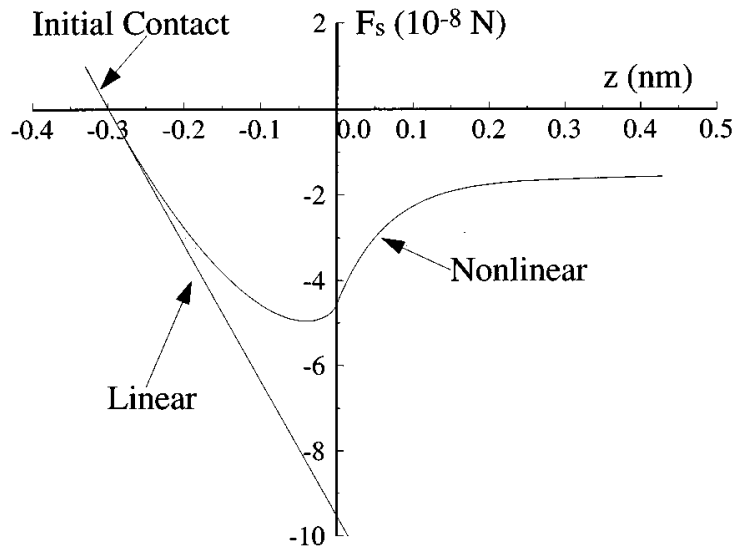

FIG. 7. Interaction force curves used for calculations. Both the linear and nonlinear curves are shown. The straight line is tangent to the nonlinear curve at the point of initial contact between the tip and surface.

In Eqs. (24), the reduced elastic modulus, $E^{*}$, is written in terms of the elastic moduli of the tip and surface, $E_{T}$ and $E_{S}$, and Poisson's ratio of the tip and surface, $\nu_{T}$ and $\nu_{S}$, as

$$
\frac{1}{E^{*}}=\frac{1-\nu_{T}^{2}}{E_{T}}+\frac{1-\nu_{S}^{2}}{E_{S}} \text {. }
$$

$R$ is the tip radius, $\gamma_{S O}$, is the adhesion constant between the tip and the sample surface, $\alpha$ is the inverse of the shielding length of the metallic adhesion, $\gamma_{w}$ is the adhesion constant between two water films, and $d$ is the thickness of the water film. The constants $C_{1}$ and $C_{2}$ as well as the point $z_{a}$ are determined by enforcing continuity of the position and slope between the functions $F_{\text {inter }}(z), F_{\mathrm{WF}}(z)$, and $F_{\text {cont }}(z)$. However, the derivative is not continuous at $z=0$. The force $F_{0}$ characterizes all forces not explicitly described by the modified Hertzian model. The following parameters, corresponding to a $\langle 100\rangle$ single crystal silicon tip interacting with a chromium surface, ${ }^{21}$ were used in the numerical results that follow:

$$
\begin{aligned}
& R=20 \mathrm{~nm}, \quad \gamma_{S O}=0.167 \mathrm{~N} / \mathrm{M}, \quad \alpha=15 \mathrm{~nm}^{-1}, \\
& F_{0}=-0.456 \mathrm{nN}, \\
& E_{T}=130 \mathrm{Gpa}, \quad \nu_{T}=0.181, \quad E_{S}=204 \mathrm{GPa}, \\
& \nu_{S}=0.26, \\
& \gamma_{w}=0.072 \mathrm{~N} / \mathrm{m}, \quad d=2.83 \mathrm{~nm} .
\end{aligned}
$$

The region near the transition between the above force models is shown in Fig. 7. The straight line is a linear approximation which is tangent at the equilibrium position. The slope of this line is the value of the linear spring stiffness which is used for comparison with the nonlinear results.

In the following simulations, the beam is assumed to be initially in contact with the specimen surface. In this case, the initial beam deflection, which can be measured experimentally, must balance with the interaction forces such that

$$
k_{\mathrm{FMA}} y(L, t=0)=F_{s}\left[y(L, t=0)-y_{0}\right] .
$$

Equation (27) can be solved iteratively for the quantity $y_{0}$ defining the center of action of the forces. Using the pa- rameters for the interaction forces, Eqs. (26), and the parameters for the beam given above along with a measured initial beam deflection of $y(L, t=0)=8.63 \mathrm{~nm}$ we find that $y_{0}$ $=8.9404 \mathrm{~nm}$.

The above interaction force model is used in the following section to demonstrate the nonlinear vibrations of the elastic cantilever. When the specimen surface is vibrated, the forcing is given by $F_{s}\left[y(L, t)-y_{0}-a_{2}(t)\right]$.

\section{B. Frequency response}

The nonlinear response of the vibrating cantilever was calculated using the finite difference method discussed in the Appendix. Solutions for both the elastic beam and the firstmode approximation are presented for comparison. The interaction force curve discussed in Sec. IV A changes very quickly near $z=0$. Therefore, the change in displacement at each time step was monitored to insure that the change in displacement was small compared to the length scale over which the interaction force curve changes quickly. For the examples presented here, the maximum change in amplitude at any time step ( $\Delta t=0.01 \mathrm{~ns})$ was always less than $1.13 \times 10^{-4} \mathrm{~nm}$ which was judged suitable for the interaction force curve shown in Fig. 7. The numerical stability of the solutions presented here was also checked.

The same values of damping were used for all examples presented here. The system damping was chosen such that the first mode of the uncoupled beam had a $Q$ of 10 . Thus,

$$
\frac{\gamma}{\rho A}=2 \beta_{\mathrm{FMA}}=\frac{\omega_{1}}{10} .
$$

The interaction damping was chosen to be 10 times this value of damping or

$$
\frac{\sigma_{\text {int }}}{\rho A L}=\omega_{1} .
$$

The frequency response of the vibrating beam with the nonlinear boundary condition is first examined so that several relevant frequencies can be chosen for the time dependent examination. The beam was initially in contact with the specimen surface and deflected according to the force balance governed by Eq. (2) above. The specimen surface was excited with $\Omega_{2}=1.6 \mathrm{MHz}$, a frequency that lies between the two resonances of the linear response of 1.33 and 1.99 $\mathrm{MHz}$. The amplitude was ramped from zero to $0.5 \mathrm{~nm}$ in 20 cycles and then held constant for $500 \mu \mathrm{s}$. The time domain response of the end of the beam was then fast Fourier transformed and squared. The power spectra from this steadystate excitation for the elastic beam solution and the firstmode approximation are shown in Fig. 8. The vertical dashed lines correspond to the natural frequencies of the beam vibrations when attached to a linear spring with appropriate stiffness calculated from the slope of the interaction force curve at the equilibrium position (see Fig. 7).

The power spectra for the two solutions are extremely different. The spectrum calculated from the FMA does have distinct nonlinear traits as expected. There are peaks, or strong responses, at multiples and subharmonics of the excitation frequency as has been observed in experiments. ${ }^{6,10}$ 

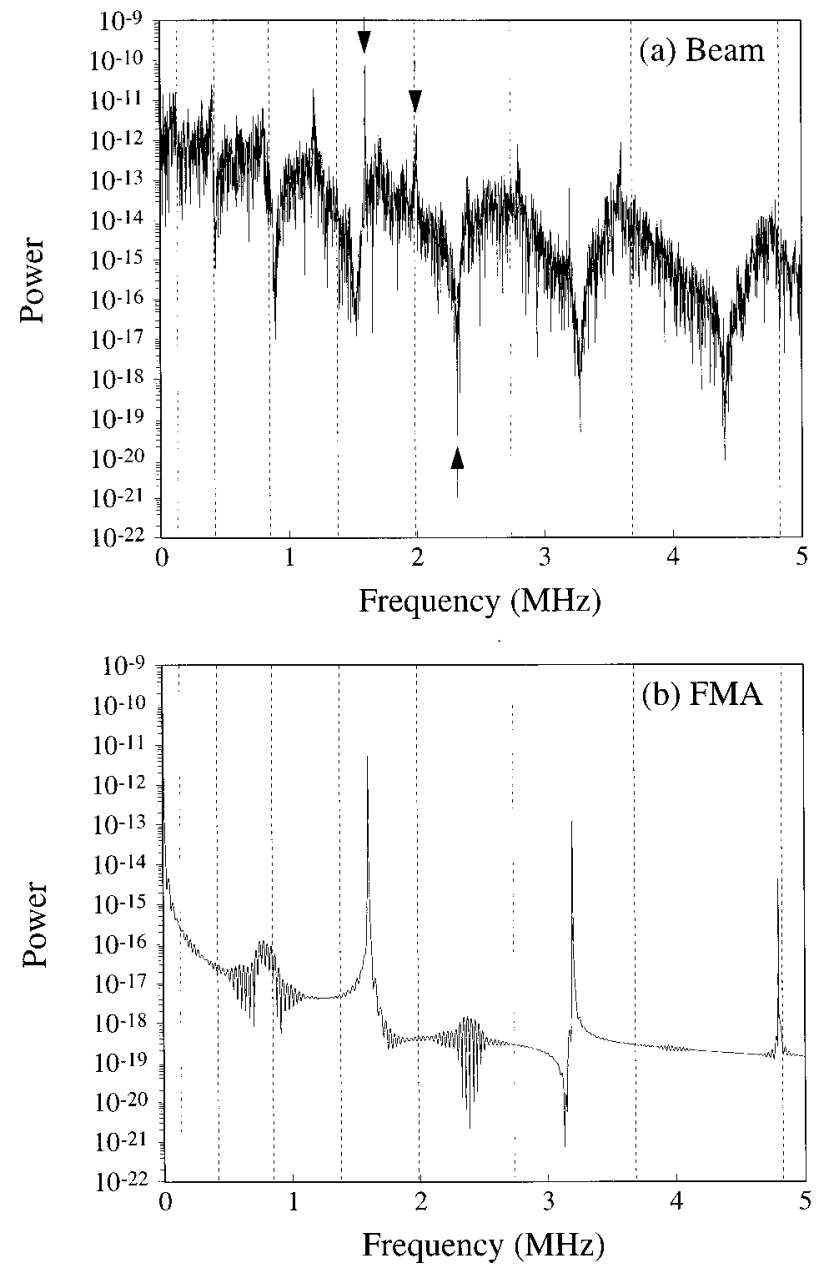

FIG. 8. Spectral response of the two dynamical systems considered here when the interaction forces are modeled nonlinearly: (a) spectrum of the motion of the end of the elastic beam, (b) spectrum of the first-mode approximation. The arrows in (a) indicate the frequencies at which the temporal response of both systems is examined. The vertical dashed lines are the natural frequencies of the beam calculated from the linear approximation of the interaction force curve at the initial contact (see Fig. 7).

These responses are clearly seen at $\Omega_{2} / 2, \Omega_{2}, 3 \Omega_{2} / 2$, $2 \Omega_{2}, 5 \Omega_{2} / 2$, and $3 \Omega_{2}$. The oscillations seen in Fig. $8(\mathrm{~b})$ have a period corresponding to the first-mode resonance. Thus, nonlinear mixing between the first resonance and the excitation frequency is observed. However, aside from these responses the frequency response has no other noticeable features. The power spectrum for the elastic beam solution is, on the other hand, very complex and covers a wide frequency range even though the excitation was at a single frequency. We see a large degree of mixing between the various modes and the excitation frequency. A similar type of frequency response has been observed in experiments. ${ }^{6,22}$ Such behavior is not captured by the FMA.

The nonlinearity also acts to shift the natural frequencies. The peaks in the power spectrum are near the peaks calculated from the linear spring, but some have been shifted higher and some have been shifted lower in frequency. The nonlinear effects act to stiffen some modes while softening others. This may help explain the failure of linear models to predict the natural frequencies measured in experiments. ${ }^{5}$

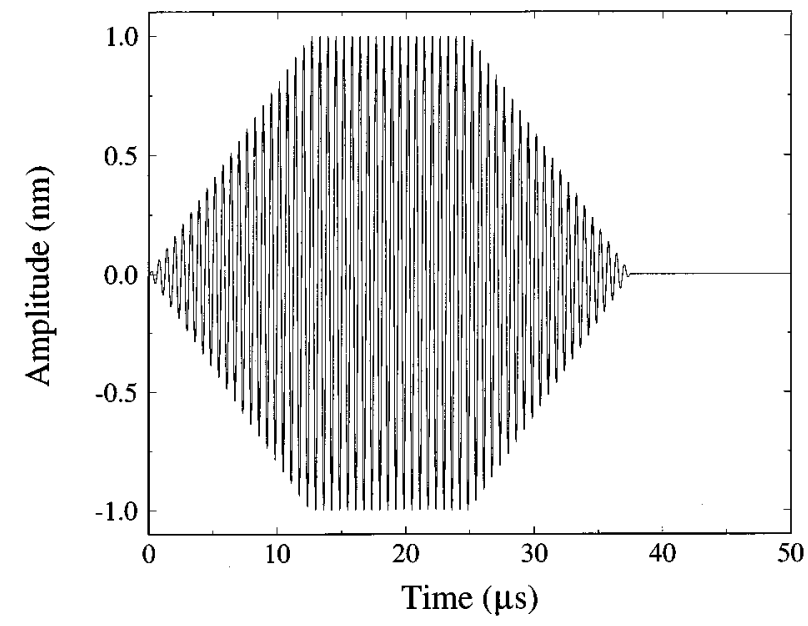

FIG. 9. Surface amplitude, $a_{2}(t)$, used for comparing the two dynamical systems. The amplitude is ramped from 0 to $1 \mathrm{~nm}$ in 20 cycles, held constant for 20 cycles, and ramped to zero in 20 cycles. The excitation frequency shown here is $\Omega_{2}=1.6 \mathrm{MHz}$.

The spread in frequencies however, is caused not only by the damping, but also by the nonlinearity. The temporal response of these systems is now examined at several different frequencies.

\section{Temporal response}

The temporal response of the elastic beam and the firstmode approximation can now be compared for a variety of frequencies based upon the results shown in Fig. 8. The responses at 1.6, 2.0, and $2.32 \mathrm{MHz}$ indicated by arrows in Fig. 8(a), are presented here. As seen from Fig. 8(a), these frequencies correspond to a nonresonance (moderate response), a resonance (large response), and an antiresonance (low response), respectively. Thus, the ability of the FMA to model the elastic beam response for excitations above the first resonance will be fully tested.

The specimen surface was first excited by a variable amplitude harmonic wave with $\Omega_{2}=1.6 \mathrm{MHz}$. The excitation was ramped linearly in 20 cycles $(12.5 \mu \mathrm{s})$, held at constant amplitude of $1 \mathrm{~nm}$ for 20 cycles, and linearly ramped back to zero in 20 cycles. Total excitation time was $37.5 \mu \mathrm{s}$. The specimen excitation function, $a_{2}(t)$, is shown in Fig. 9. The results are shown in Figs. 10(a) and 10(b) for the elastic beam solution and the first-mode approximation, respectively. The responses are qualitatively as well as quantitatively different. The elastic beam solution has many more high-frequency components at higher amplitudes than the FMA result. This result is not such a surprise since the higher modes are neglected by the FMA. The different response caused by the interaction damping is also observed in Fig. 10. Certain higher frequency modes are not damped to the same extent as other modes.

The mean response from both solutions can also be compared. The above time domain responses were low-pass filtered to give the mean response. This result is shown in Fig. 11. Here it is clear that the FMA has also a different response than that of the elastic beam solution. The FMA solution is very symmetric in time, reaching its maximum in phase with the excitation. The elastic beam solution has a slight time lag 


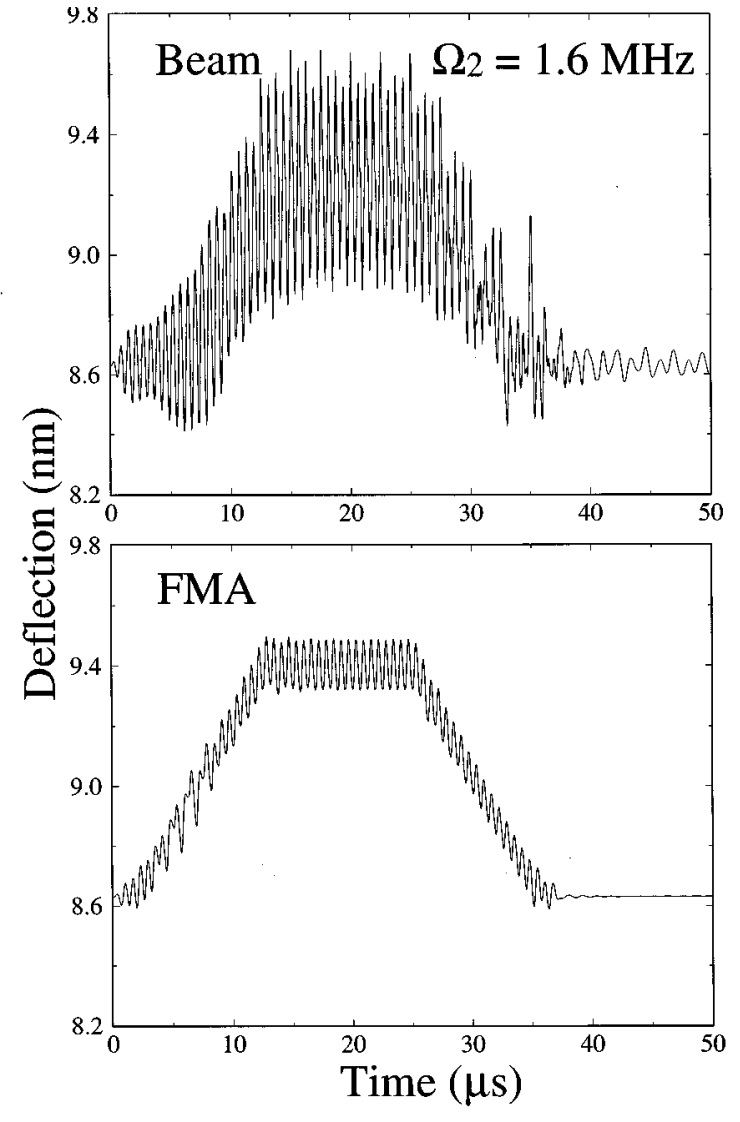

FIG. 10. Time domain response of the two models to the surface excitation shown in Fig. 9 at the nonresonant frequency of $\Omega_{2}=1.6 \mathrm{MHz}$. The system damping and interaction damping are given by $\gamma / \rho A=2 \beta_{\mathrm{FMA}}=\omega_{1} / 10$ and $\sigma_{\text {int }} / \rho A L=\omega_{1}$, respectively.

before reaching its maximum and is not as symmetric as the FMA solution. Both of these characteristics have been observed in experiments with high-frequency excitations of atomic-force microscope cantilevers. ${ }^{2,6,10,17,22}$ Similar results

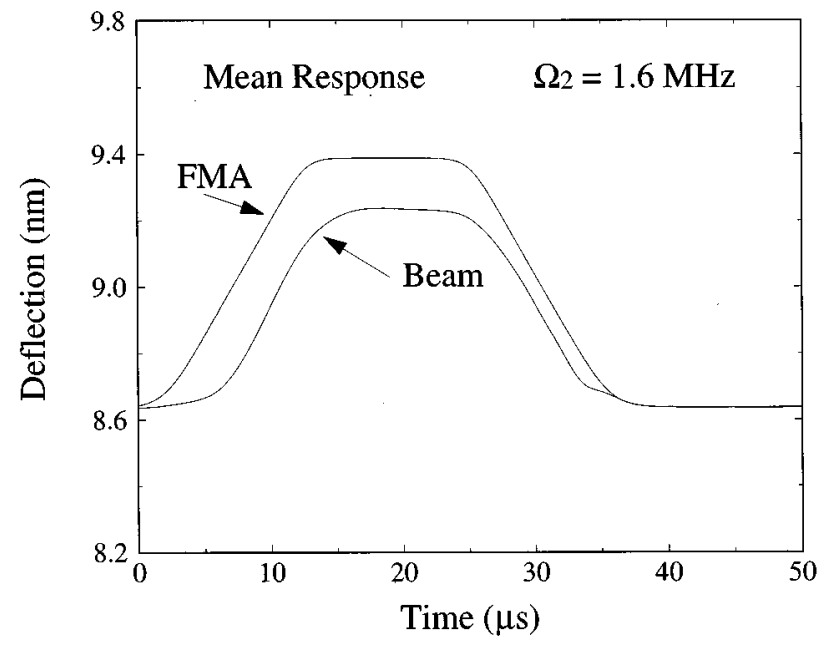

FIG. 11. Time domain signals shown in Fig. 10 after being low-pass filtered. The solution from the first-mode approximation is very symmetric and rises to a much higher amplitude than the more asymmetric solution of the elastic beam.

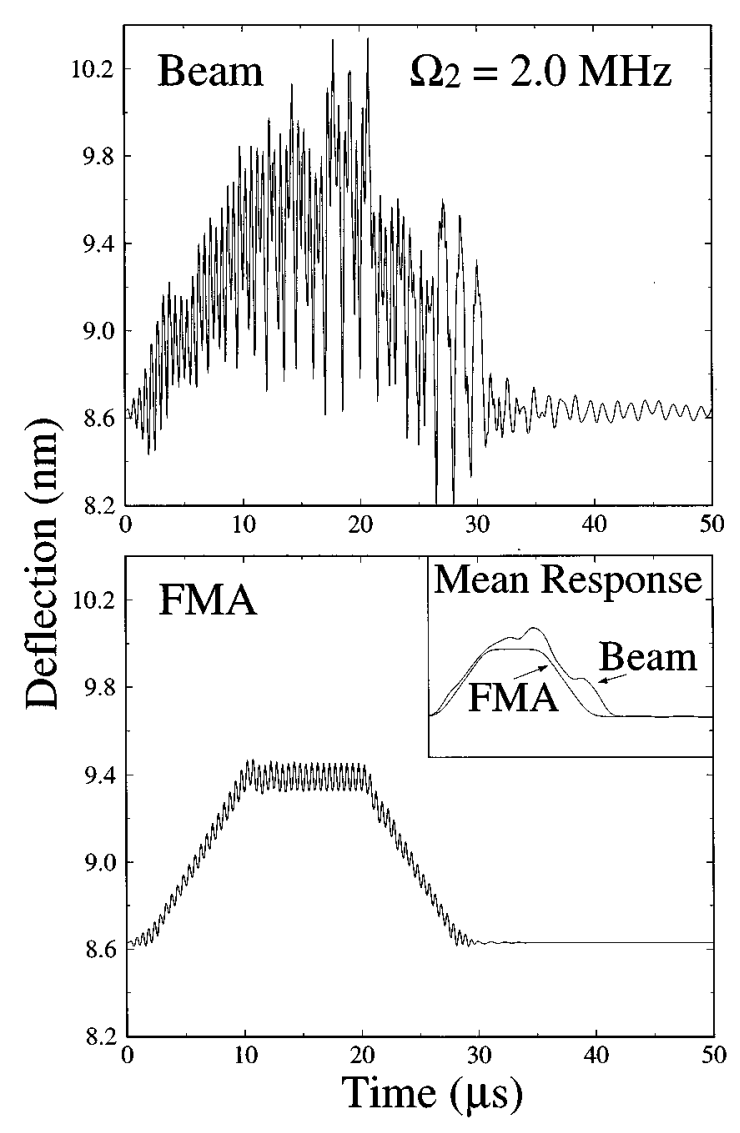

FIG. 12. Time domain response of the two models to the surface excitation similar to that shown in Fig. 9 at a resonance frequency of $\Omega_{2}=2.0 \mathrm{MHz}$. The damping is the same as in Fig. 10. The inset shows the comparison of the low-pass filtered results of the two models.

have also been obtained using a different solution technique. ${ }^{21}$ The low-frequency response is seen to be influenced by the complex mixing of the higher modes as well as by the interaction damping.

The resonant frequency of $\Omega_{2}=2.0 \mathrm{MHz}$ was examined next. The excitation was ramped linearly in 20 cycles (10 $\mu \mathrm{s})$, held at a constant amplitude of $1 \mathrm{~nm}$ for 20 cycles, and linearly ramped back to zero in 20 cycles. Total excitation time was $30 \mu$ s. The results are shown in Fig. 12 for the elastic beam solution and the first-mode approximation. As expected, the FMA solution is worse than the previous nonresonant case, although not tremendously so. The high level of damping prevents the elastic beam resonance from having an extremely high response. The inset shows the comparison of the low-pass filtered results of both solutions.

Finally, the antiresonant frequency of $\Omega_{2}=2.32 \mathrm{MHz}$ was examined. The excitation was ramped linearly in 30 cycles $(13 \mu \mathrm{s})$, held at a constant amplitude of $1 \mathrm{~nm}$ for 30 cycles, and linearly ramped back to zero in 30 cycles. Total excitation time was $39 \mu$ s. The results are shown in Fig. 13 for the elastic beam solution and the first-mode approximation. As expected, the FMA does a better job of matching this response, especially the low-frequency behavior. The low-pass filtered responses are again shown in the inset. As seen in Fig. 8, the response at this frequency is expected to 


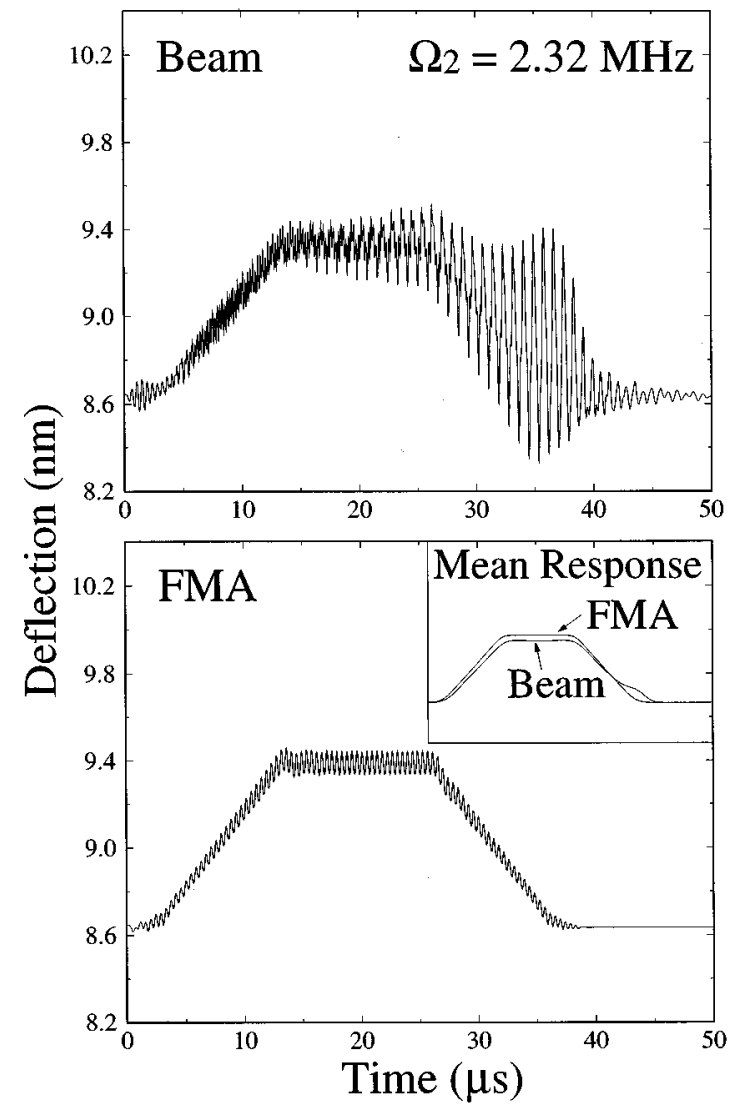

FIG. 13. Time domain response of the two models to the surface excitation similar to that shown in Fig. 9 at an antiresonance frequency of $\Omega_{2}=$ 2.32 MHz. The damping is the same as in Fig. 10. The low-pass filtered responses of the two models are shown in the inset.

be much smaller than at other frequencies. This fact helps the FMA better match the low-frequency response. However, as before, the two results are quantitatively different.

As expected the FMA performed better at nonresonant frequencies than at resonant frequencies. The resonances are generally not known before an experiment is made and they can be difficult to find experimentally. Therefore, the nonresonant result shown in Fig. 10 is probably most representative of the ability of the FMA to model the response of atomic-force microscope cantilevers which are excited above the first resonance frequency. The high-frequency response of the beam was underestimated by the FMA for all frequencies observed. The low-frequency response predicted by the FMA was qualitatively similar to the low-frequency response of the elastic beam. However, the two solutions had quantitative differences that may be important when comparison with experiments is made. Because the FMA is a lowfrequency approximation, its use for modeling excitation frequencies above the first natural frequency must be made with caution.

\section{DISCUSSION}

The high-frequency responses of atomic-force microscope cantilevers were examined here in detail and compared with solutions using a first-mode approximation. Two impor- tant differences in the linear behavior of these two dynamical systems were discussed. The drive-point impedance and the effects of interaction damping are distinctly different in these two systems. These differences were shown to be important whenever the excitation frequency is above the natural frequency of the first flexural mode. Therefore, caution must be exercised when using low-frequency approximations such as the FMA to model the high-frequency dynamics of these beams.

A direct numerical simulation of the elastic beam equation using a finite difference approximation was also presented. These simulations provided insight into the importance of the higher-order modes. It was shown that the higher frequency modes are very important when an accurate view of the dynamics is needed. It was also shown that the lowfrequency response to a high-frequency excitation required the inclusion of the higher modes. Although inclusion of these higher order modes complicates the problem considerably, it is indeed a necessity governed by the complex dynamics. Numerical solutions are often the final step in solving these types of nonlinear problems. Therefore inclusion of all modes should not be prohibitive. The finite difference method used here is very efficient and provides the high level of accuracy needed for comparisons with experimental results. The deflection of all points along the beam are calculated simultaneously which may also be useful for comparison with experimental results. It is anticipated that numerical simulation techniques will allow further progress to be made in understanding the complex nonlinear interaction forces that affect the motion of atomic-force microscope cantilevers. Differences in expected vibrational response can easily be calculated for a number of different forceinteraction models, as for instance, those with hysteretic behavior, for comparison.

One other note should be also made about the calculations and nonlinear responses observed. A chaotic type of motion was observed in various simulations when the excitation amplitude was large. The beam was observed to jump from contact mode to free vibration mode in a chaotic fashion. The numerical stability of these solutions is still being examined. Such chaotic responses, which have also been observed experimentally, ${ }^{10,17,23}$ are highly sensitive to slight changes in initial conditions and boundary conditions. Therefore, experimental repeatability, which is important for measurement of material properties or surface properties, is nearly impossible for this type of motion. Although the chaotic behavior is interesting from a nonlinear dynamics point of view, its use for quantitative measurements is, for this reason, questioned.

\section{ACKNOWLEDGMENTS}

The Alexander von Humboldt Foundation is gratefully acknowledged for their support of one of the authors (J.A.T.) during the course of this work. This research was also partially supported by grants from the Volkswagen Foundation and the European Union within the Human Capital Mobility program. 


\section{APPENDIX}

Solutions of the partial differential equations, Eqs. (1) and (5), with nonlinear boundary conditions are not trivial. A numerical approach is used in many of the examples presented above. A direct numerical simulation, such as the finite difference approximation discussed here, has a number of advantages. An efficient numerical solution allows the problem to be solved for a large parameter range so that the vibration dependence can be observed. It is then possible for new experiments to be suggested in which certain interaction parameters can be better observed.

The finite difference approximation is a standard numerical technique used to solve differential equations. This technique is discussed in a number of excellent texts and will not be discussed in detail here. ${ }^{15,16}$ The derivatives in the governing equations are, in essence, approximated by a Taylor series expansion. The beam is divided into a number of finite segments of size $\Delta x$ such that $x=n \Delta x$. The motion of each segment is related to the motion of the neighboring segments. Time is also discretized such that $t=j \Delta t$. In the following discussion, the subscripts to $y$ refer to the temporal step and the superscripts refer to the spatial nodes.

The second order, centered finite difference approximations for spatial derivatives are given by

$y^{\prime}(x, t) \cong \frac{y_{j}^{(n+1)}-y_{j}^{(n-1)}}{2 \Delta x}$,

$y^{\prime \prime}(x, t) \cong \frac{y_{j}^{(n+1)}-2 y_{j}^{(n)}+y_{j}^{(n-1)}}{(\Delta x)^{2}}$,

$y^{\prime \prime \prime}(x, t) \cong \frac{y_{j}^{(n+2)}-2 y_{j}^{(n+1)}+2 y_{j}^{(n-1)}-y_{j}^{(n-2)}}{2(\Delta x)^{3}}$,

$y^{\prime \prime \prime \prime}(x, t) \cong \frac{y_{j}^{(n+2)}-4 y_{j}^{(n+1)}+6 y_{j}^{(n)}-4 y_{j}^{(n-1)}+y_{j}^{(n-2)}}{(\Delta x)^{4}}$

The second order, centered finite difference approximations for the necessary temporal derivatives are

$$
\begin{aligned}
& \dot{y}(x, t) \cong \frac{y_{j+1}^{(n)}-y_{j-1}^{(n)}}{2 \Delta t}, \\
& \ddot{y}(x, t) \cong \frac{y_{j+1}^{(n)}-2 y_{j}^{(n)}+y_{j-1}^{(n)}}{(\Delta t)^{2}} .
\end{aligned}
$$

The discretized equation of motion for the elastic beam, Eq. (1), is then approximated as

$$
\frac{E I}{(\Delta x)^{4}}\left(y_{j}^{(n+2)}-4 y_{j}^{(n+1)}+6 y_{j}^{(n)}-4 y_{j}^{(n-1)}+y_{j}^{(n-2)}\right)
$$

$$
+\frac{\gamma}{2 \Delta t}\left(y_{j+1}^{(n)}-y_{j-1}^{(n)}\right)+\frac{\rho A}{(\Delta t)^{2}}\left(y_{j+1}^{(n)}-2 y_{j}^{(n)}+y_{j-1}^{(n)}\right)=0 \text {. }
$$

Equation (A3) can be rearranged to yield a time-stepping equation

$$
\begin{aligned}
y_{j+1}^{(n)}= & \left(\frac{1}{1+C}\right)\left\{2 y_{j}^{(n)}-(1-C) y_{j-1}^{(n)}\right. \\
& \left.-D\left[y_{j}^{(n+2)}-4 y_{j}^{(n+1)}+6 y_{j}^{(n)}-4 y_{j}^{(n-1)}+y_{j}^{(n-2)}\right]\right\},
\end{aligned}
$$

where the constants $D$ and $C$ are given by

$$
D=\frac{E I}{\rho A} \frac{(\Delta t)^{2}}{(\Delta x)^{4}}, \quad C=\frac{\gamma}{\rho A} \frac{\Delta t}{2} .
$$

Thus, the motion at the next step in time is determined by the two previous time steps. Discretized versions of the boundary conditions, Eqs. (3) and (4), are given by

$$
\begin{aligned}
& y_{j}^{(0)}=A_{1} \sin \left(\Omega_{1} j \Delta t\right), \\
& y_{j}^{(1)}=y_{j}^{(-1)}, \\
& y_{j}^{(N+1)}=2 y_{j}^{(N)}-y_{j}^{(N-1)}, \\
& y_{j}^{(N+2)}=2 y_{j}^{(N+1)}-2 y_{j}^{(N-1)}+y_{j}^{(N-2)} \\
& -\left(\frac{2(\Delta x)^{3}}{E I}\right)\left[F_{s}\left[y_{j}^{(N)}, a_{2}(j \Delta t)\right]\right. \\
& \left.-\sigma_{\text {int }}\left(\frac{y_{j+1}^{(N)}-y_{j-1}^{(N)}}{2 \Delta t}-\dot{a}_{2}(j \Delta t)\right)\right] \text {. }
\end{aligned}
$$

The above boundary conditions contain three terms that are not actual locations on the beam, $y_{j}^{(-1)}, y_{j}^{(N+1)}$, and $y_{j}^{(N+2)}$. These "fictitious" nodes are required for the time stepping equations defining the nodes near the ends of the beam. We see that Eq. (A3) for nodes $n=1, n=N-1$, and $n=N$ is given by

$$
\begin{aligned}
y_{j+1}^{(1)}= & \left(\frac{1}{1+C}\right)\left\{2 y_{j}^{(1)}-(1-C) y_{j-1}^{(1)}\right. \\
& \left.-D\left[y_{j}^{(3)}-4 y_{j}^{(2)}+6 y_{j}^{(1)}-4 y_{j}^{(0)}+y_{j}^{(-1)}\right]\right\},
\end{aligned}
$$

$$
\begin{aligned}
y_{j+1}^{(N-1)}= & \left(\frac{1}{1+C}\right)\left\{2 y_{j}^{(N-1)}-(1-C) y_{j-1}^{(N-1)}-D\left[y_{j}^{(N+1)}\right.\right. \\
& \left.\left.-4 y_{j}^{(N)}+6 y_{j}^{(N-1)}-4 y_{j}^{(N-2)}+y_{j}^{(N-3)}\right]\right\}, \quad(\mathrm{A} 7)
\end{aligned}
$$




$$
\begin{aligned}
y_{j+1}^{(N)}= & \left(\frac{1}{1+C}\right)\left\{2 y_{j}^{(N)}-(1-C) y_{j-1}^{(N)}-D\left[y_{j}^{(N+2)}\right.\right. \\
& \left.\left.-4 y_{j}^{(N+1)}+6 y_{j}^{(N)}-4 y_{j}^{(N-1)}+y_{j}^{(N-2)}\right]\right\} .
\end{aligned}
$$

The boundary conditions given in Eqs. (A6) allow the above equations to be written in terms of the "real" nodes as

$$
\begin{aligned}
& y_{j+1}^{(1)}=\left(\frac{1}{1+C}\right)\left\{2 y_{j}^{(1)}-(1-C) y_{j-1}^{(1)}-D\left[y_{j}^{(3)}-4 y_{j}^{(2)}\right.\right. \\
& \left.\left.+7 y_{j}^{(1)}-4 A_{1} \sin \left(j \Delta t \Omega_{1}\right)\right]\right\}, \\
& y_{j+1}^{(N-1)}=\left(\frac{1}{1+C}\right)\left\{2 y_{j}^{(N-1)}-(1-C) y_{j-1}^{(N-1)}-D\left[-2 y_{j}^{(N)}\right.\right. \\
& \left.\left.+5 y_{j}^{(N-1)}-4 y_{j}^{(N-2)}+y_{j}^{(N-3)}\right]\right\}, \\
& y_{j+1}^{(N)}=\left(\frac{1}{1+C+\frac{\sigma_{\text {int }} \Delta t}{\rho \Delta x}}\right)\left(2 y_{j}^{(N)}-(1-C) y_{j-1}^{(N)}\right. \\
& -D\left\{2 y_{j}^{(N)}-4 y_{j}^{(N-1)}+2 y_{j}^{(N-2)}-\frac{2(\Delta x)^{3}}{E I}\right. \\
& \times\left[F_{s}\left[y_{j}^{(N)}, a_{2}(j \Delta t)\right]+\sigma_{\mathrm{int}}\left(\frac{y_{j-1}^{(N)}}{2 \Delta t}\right.\right. \\
& \left.\left.\left.\left.+\dot{a}_{2}(j \Delta t)\right)\right]\right\}\right)
\end{aligned}
$$

Similar equations can also be written for the other nodes. An equation for the motion of the $n=0$ node is not required since its motion is given directly by the boundary condition in Eqs. (A6).

The initial conditions, given by Eq. (2), are now written

$$
y_{0}^{(n)}=y_{-1}^{(n)}=-\frac{F_{0}}{6 E I}(n \Delta x)^{2}(n \Delta x-3 L) .
$$

A new vector can then be defined that contains all of the node locations at a particular time step $j$. This vector, of length $N$, is given by

$$
\underline{Y}_{j}=\left\{\begin{array}{c}
y_{j}^{(1)} \\
y_{j}^{(2)} \\
y_{j}^{(3)} \\
\cdot \\
\cdot \\
\cdot \\
y_{j}^{(N-2)} \\
y_{j}^{(N-1)} \\
y_{j}^{(N)}
\end{array}\right\} .
$$

The finite difference progression is then written in terms of this vector by

$$
\begin{aligned}
\underline{Y}_{j+1}= & \left(\frac{1}{1+C+\delta_{n N} \frac{\sigma_{\mathrm{int}} \Delta t}{\rho A \Delta x}}\right) \\
& \times\left[2 \underline{Y}_{j}-(1-C) \underline{Y}_{j-1}-D \underline{M}_{\underline{Y}}-D \underline{G}\right],
\end{aligned}
$$

where the symbol $\delta_{n N}$ implies that this term is included only when $n=N$. The $N \times N$ matrix $M$ and forcing vector $\underline{G}$ of length $N$ are given by

$$
M=\left[\begin{array}{cccccccc}
7 & -4 & 1 & 0 & \cdot & . & \cdot & \cdot \\
-4 & 6 & -4 & 1 & 0 & . & \cdot & \cdot \\
1 & -4 & 6 & -4 & 1 & 0 & \cdot & \cdot \\
0 & \cdot & . & . & . & . & 0 & \cdot \\
\cdot & 0 & 1 & -4 & 6 & -4 & 1 & 0 \\
\cdot & . & 0 & 1 & -4 & 6 & -4 & 1 \\
\cdot & \cdot & \cdot & 0 & 1 & -4 & 5 & -2 \\
\cdot & \cdot & \cdot & \cdot & 0 & 2 & -4 & 2
\end{array}\right],
$$

$$
G=\left\{\begin{array}{c}
-4 A_{1} \sin \left(\Omega_{1} j \Delta t\right) \\
A_{1} \sin \left(\Omega_{1} j \Delta t\right) \\
0 \\
0 \\
\cdot \\
\cdot \\
0 \\
-\frac{2(\Delta x)^{3}}{E I}\left[F_{s}\left[y_{j}^{(N)}, a_{2}(j \Delta t)\right]+\sigma_{\mathrm{int}}\left(\frac{y_{j-1}^{(N)}}{2 \Delta t}+\dot{a}_{2}(j \Delta t)\right)\right]
\end{array}\right\} .
$$


The width of the beam segments, $\Delta x$, must be chosen small enough such that the segment length is several times smaller than the wavelength of the highest mode to be described. This aspect is discussed in more detail in the text of the article. The stability criterion for the numerical progression requires that $D<1 .{ }^{15,16}$ This implies a limitation on the time step that $\Delta t<(\Delta x)^{2} \sqrt{E I / \rho A}$.

A similar finite difference approximation can also be made for the first-mode approximation given by Eq. (5). Using the above approximations, the time-stepping equation for the FMA is

$$
\begin{aligned}
d_{j+1}= & \left(\frac{1}{1+\beta_{\mathrm{FMA}} \Delta t+\frac{\sigma_{\mathrm{int}} \Delta t}{2 m_{\mathrm{FMA}}}}\right)\left\{\left[2-\frac{k_{\mathrm{FMA}}}{m_{\mathrm{FMA}}}(\Delta t)^{2}\right] d_{j}\right. \\
& -\left[1-\beta_{\mathrm{FMA}} \Delta t\right] d_{j-1}+\frac{(\Delta t)^{2}}{m_{\mathrm{FMA}}}\left[F_{s}\left[d_{j}, a_{2}(j \Delta t)\right]\right. \\
& \left.\left.+\sigma_{\mathrm{int}}\left(\frac{d_{j-1}}{2 \Delta t}+\dot{a}_{2}(j \Delta t)\right)\right]\right\} .
\end{aligned}
$$

These finite difference solutions to the governing equations of motion were used for the numerical solutions presented above.

${ }^{1}$ G. Binning, C. F. Quate, and C. Gerber, Phys. Rev. Lett. 56, 930 (1986).

${ }^{2}$ U. Rabe and W. Arnold, Appl. Phys. Lett. 64, 1493 (1994).

${ }^{3}$ K. Yamanaka and S. Nakano, Jpn. J. Appl. Phys. 35, 3787 (1996).
${ }^{4}$ S. R. Manalis, S. C. Minne, A. Atalar, and C. F. Quate, Rev. Sci. Instrum. 67, 3294 (1996).

${ }^{5}$ U. Rabe, K. Janser, and W. Arnold, Rev. Sci. Instrum. 67, 3281 (1996).

${ }^{6}$ U. Rabe, Ph.D. dissertation, Universität des Saarlandes, 1996.

${ }^{7}$ S. Hirsekorn, U. Rabe, and W. Arnold, Europhys. News 27, 93 (1996).

${ }^{8}$ E. L. Florin, M. Radmacher, B. Fleck, and H. E. Gaub, Rev. Sci. Instrum. 65, 639 (1994).

${ }^{9}$ N. A. Burnham, G. Gremaud, A. J. Kulik, P.-J. Gallo, and F. Oulevey, J. Vac. Sci. Technol. B 14, 1308 (1996).

${ }^{10}$ N. A. Burnham, A. J. Kulik, G. Gremaud, P.-J. Gallo, and F. Oulevey, J. Vac. Sci. Technol. B 14, 794 (1996).

${ }^{11}$ L. Meirovitch, Analytical Methods in Vibrations (Macmillan, New York, 1967).

${ }^{12}$ M. C. Junger and D. Feit, Sound Structures, and Their Interaction (Acoustical Society of America, Woodbury, NY, 1993).

${ }^{13}$ The smoothed trend of the impedance is proportional to the density of modes of the system divided by $\omega$. The density of modes in this case is proportional to $\partial k / \partial \omega$ which increases with frequency as $\sqrt{\omega}$.

${ }^{14}$ Mathematica, A System for Doing Mathematics by Computer (Wolfram Research Inc., 100 Trade Center, Champaign, IL, 1991).

${ }^{15}$ D. Greenspan, Discrete Numerical Methods in Physics and Engineering (Academic, New York, 1974).

${ }^{16}$ G. F. Carrier and C. E. Pearson, Partial Differential Equations, Theory and Technique (Academic, New York, 1976).

${ }^{17}$ N. A. Burnham, A. J. Kulik, G. Gremaud, and G. A. D. Briggs, Phys. Rev. Lett. 74, 5092 (1995).

${ }^{18}$ K. Bodlund, J. Sound Vib. 73, 19 (1987).

${ }^{19}$ F. Kawakami and K. Yamiguchi, J. Acoust. Soc. Am. 80, 543 (1986).

${ }^{20}$ M. R. Schroeder, Some New Results in Reverberation Theory, Proceedings of the 5th International Congress in Acoustics, (Elsevier, Amsterdam, 1965), Vol. 1b, G31, 1965, pp. 1-4.

${ }^{21}$ S. Hirsekorn, U. Rabe, and W. Arnold, Nanotechnology 8, 57 (1997).

${ }^{22}$ P.-E. Mazeran and J.-L. Loubet, Tribol. Lett. 3, 125 (1997).

${ }^{23}$ U. Rabe, M. Dvorak, and W. Arnold, Thin Solid Films 264, 165 (1995). 